\title{
Hyperattenuated Intracerebral Lesions after Mechanical Recanalization in Acute Stroke: Contrast and Compare
}

L ummel et $\mathrm{al}^{1}$ herein report their observations regarding hyperdense cerebral lesions (HCL) identified after mechanical revascularization in acute ischemic stroke. They construct a nexus between HCL and iodinated radiographic contrast material (IRCM) deposition, which may either disappear within 24 hours (enhancement) or persist as intracerebral hemorrhagic transformation lesions (HL). They analyzed the relationship of HCL to clinical outcome and concluded that HCL indicated a higher risk of secondary HL but that their presence does not seem to be of any prognostic value for clinical outcome.

The latter conclusion seems predicated on a comparison between 16 patients without HCL and 85 with HCL, in which no statistical difference was identified between the 2 groups in the modified Rankin Scale 0-3 outcome. A more convincing conclusion regarding functional outcome might be derived if the sample size of the 2 groups were larger or perhaps the customary mRS $0-2$ end points were analyzed. The small group of 16 patients without HCL may well have been favorably predisposed to a $93 \%$ revascularization and a $40 \% \mathrm{mRS}$ $0-3$ outcome. The subjects with HCL may have been otherwise predisposed to a poorer $74 \%$ revascularization and a $33.7 \%$ mRS $0-3$ outcome. Lack of adjustment for relevant comorbidities leaves this question unresolved.

Within this HCL group, we are left to wonder about functional outcome differences between the HCL-enhancement group $(n=41)$ and the HCL-HL group $(n=43)$. We are told that there was no significant difference. Due to the lack of specific figures, we can only speculate on what percentage difference and/or $P$ value might be sufficiently hypothesis-generating among those whose HCL disappear within 24 hours versus those in whom they persist and lead to hemorrhagic transformation. A difference of 5\% might be large enough to promote further analysis, given sufficient sample sizes.

The authors are to be recognized for the very low $\mathrm{PH}$ rate, all $\mathrm{PH}$ 1. After all, $\mathrm{PH} 1$ is insignificant, with $\mathrm{PH} 2$ the yardstick of $\mathrm{PH}$ significance, according to the European Cooperative Acute Stroke Study (ECASS) experience. But is it? In the Interventional Management of Stroke (IMS) I and II trials, symptomatic intracerebral hemorrhages (ICHs) were composed of an equal number of $\mathrm{PH} 1$ and $\mathrm{PH} 2 \mathrm{~s}$, and the 2 groups had similar mean lesion (infarct $+\mathrm{ICH}$ ) volume. ${ }^{2} \mathrm{PH} 1$ and $\mathrm{PH} 2$ status was, therefore, not defined so much by the $\mathrm{PH}$ volume, but by lesion volume, in which $\mathrm{PH}$ 1s were associated with larger infarcts and a larger denominator from which to determine the $\mathrm{PH}$ status as $>30 \%$ ( $\mathrm{PH} 2$ ) or $<30 \%$ ( $\mathrm{PH} 1$ ) lesion volume. We hypothesize that endovascular (EV) revascularization studies led to smaller lesions compared with IV treatment of major arterial occlusive lesions, leading to an equal number of symptomatic PH 1 and PH 2 values of equal mean volumes in IMS I and II, as opposed to large infarcts with a large number of $\mathrm{PH} 2 \mathrm{~s}$ in ECASS. The presence of all $\mathrm{PH} 1 \mathrm{~s}$ and no $\mathrm{PH} 2 \mathrm{~s}$ raises the following question: How large were the lesion volumes in the study of Lummel et al? ${ }^{1}$ A mean volume of $92 \mathrm{~mL}$ is reported in the HCL group. However, this volume is larger than $61.6 \mathrm{~mL}$ reported for 109 anterior circulation subjects in the Solitaire Retrospective Trial. ${ }^{3}$ Surely the $92 \mathrm{~mL}$ would be reduced if the 16 non-HCL cases were included.

This speculation leads us to other questions regarding the study. The study population was selected on the basis of good Alberta Stroke Program Early CT scores and CT perfusion, in which patients without mismatch were excluded. They were treated expeditiously with IV rtPA and EV mechanical revascularization. Nevertheless, the reperfusion rates and the percentage mRS 0-2 were relatively low compared with the Solitaire Retrospective Trial, a reasonable comparator for such retrospective analysis, in which $85 \%$ modified TICI $2 \mathrm{~b}-3$ was achieved for the ICA terminus region and M1 occlusions. ${ }^{4}$ Why should patients with favorable advanced perfusion imaging have relatively low reperfusion, with a relatively low percentage of good outcomes, with an HCL incidence at the upper end of reported ranges? Where there is convergence of numerous HCL, IRCM localization, suboptimal reperfusion rates, and low functional outcome rates, are we missing an elephant in the room?

One possible common denominator to the information above is IRCM. That the HCL incidence correlated with EV low-osmolar IRCM volume in a population that already had IV IRCM infusion for CTA supports an IRCM-ICH hypothesis. 
This is consonant with our animal work, in which increased ICH was found in a rat temporary-occlusion model with intraarterial (IA) low-osmolar iohexol (Omnipaque; GE Healthcare, Piscataway, New Jersey) injection, as opposed to saline injection, despite equal infarct edema. ${ }^{5}$

Supported by the Foundation of the American Society of Neuroradiology and Boston Scientific, Morales et $\mathrm{al}^{6}$ studied 3 groups of rats with the same model, with saline, low-osmolar iopamidol (Isovue; Bracco Diagnostics, Monroe Township, New Jersey), or iso-osmolar iodixanol (Visipaque; GE Healthcare) infused IA postocclusion. They performed $3 \mathrm{~T}$ MR imaging immediately postinfusion and at 24 hours immediately before sacrifice. On MR imaging and postmortem cut sections, the iodixanol rats had not only smaller infarct edema but also less cortical ICH. ${ }^{6} \mathrm{ICH}$ and infarct volumes were not significantly different in saline and iopamidol rats.

If IRCM A is associated with smaller infarcts and less ICH than saline or IRCM B in animals, is it too far-fetched to suggest that IRCM may have an adverse effect in human use? Could IRCM A use contribute to a higher percentage of mRS 0-2 than IA IRCM $\mathrm{B}$ in humans? IMS III was designed to give insight into the potential clinical effects of IRCM in this respect. Operators recorded contrast type and volume for later analysis. Symptomatic ICH was sufficiently infrequent in IMS III and sufficiently multifactorial that an IRCM-related smoking gun would be impossible to prove, even if it existed. It is not clear that IA IRCM use contributes to symptomatic ICH directly, independent of technical factors.

IMS III showed asymptomatic ICH to be significantly more common when maintaining combined IV rtPA-EV treatment compared with IV rtPA alone; at least it did not negate an IRCMICH hypothesis. That Lummel et $\mathrm{al}^{1}$ found a relationship between procedural IRCM volume and HCL sustains a harmful IRCM hypothesis as well. Analysis of the relationship of ICH and IRCM type and volume in IMS III has been initiated, with interesting preliminary results.

That ICH differences and mRS outcome differences might occur with isomolar-versus-low-osmolar IRCM is consistent with the findings of Morales et $\mathrm{al}^{6}$ and, furthermore, is supportive of an IRCM-HCL-ICH functional outcome hypothesis. Unfortunately, IMS III stopped prematurely, and additional opportunities to identify any effect were lost.

Lummel et $\mathrm{al}^{1}$ briefly discussed potential mechanisms of the harmful effects of IRCM on the basement membrane contributing to HCL and ICH. Effects may, in fact, be additive. Did the IRCM used contribute to blood-brain barrier disruption or apoptosis of initially viable cells? Could IRCM effects be IRCM-specific: Is there a tissue osmotic effect of smaller monomeric IRCM molecules that is reduced or overcome by larger molecular intravascular dimers at the capillary and BBB level? Did a thrombotic effect of IRCM at the precapillary level contribute to reduced perfusion and worse outcomes? Did vasospasm generated by large guide catheters in the ICA and by devices in the MCA contribute to pressure-flow alterations during IRCM guide-catheter injections, contributing to HCL and potential sequelae? Did IV IRCM prior to $\mathrm{EV}$ treatment contribute to these phenomena? Is there a target at the BBB level that can be engaged to reduce or overcome adverse effects of thrombolytic therapy associated with mechanical revascularization? ${ }^{7}$

I have been personally concerned about the relationship of IRCM and outcome for many years during the IA-thrombolysis era. With the advent of mechanical revascularization, I thought this issue would now be relegated to a lower rung on the ladder of stroke-therapy-related concerns. Reported excellent revascularization rates and outcomes would overshadow any small harmful effect that IRCM might add. Identifying a less-harmful effect of one IRCM versus another may be a more reasonable option. Clearly, IRCM is not the primary determinant of EV outcomes. Nevertheless, an effect in 3\%-5\% of subjects could mean the difference between success and futility in a trial. Lummel et al ${ }^{1}$ leave me with the impression that there is still much to understand about IRCM and their use.

\section{REFERENCES}

1. Lummel N, Schulte-Altedorneburg G, Bernau C. Hyperattenuated intracerebral lesions after mechanical recanalization in acute stroke. AJNR Am J Neuroradiol 2014;35:345-51

2. Tao H, Carrozzella J, Broderick J, et al, for the IMS Investigators. Parenchymal hematoma and total lesion volume in combined IV/IA revascularization stroke therapy. I Neurointerv Surg 2012;4:256-60

3. Javadi A, Carrozzella J, Tomsick T, for the IMS I/II \& Solitaire Investigators. Lesion and ICH volume: IMS I/II vs. Solitaire study. In: Proceedings of the Ninth Annual Meeting of the Society of Neurointerventional Surgery, San Diego, California. July 23-27, 2012

4. Dávalos A, Pereira VM, Chapot R, et al. Retrospective multicenter study of Solitaire FR for revascularization in the treatment of acute ischemic stroke. Stroke 2012;43:2699-705

5. Kurosawa $\mathrm{Y}$, Lu A, Khatri $\mathrm{P}$, et al. Intra-arterial iodinated radiographic contrast material injection and intravenous heparin administration in a rat middle cerebral artery occlusion and reperfusion model: possible effects on intracerebral hemorrhage. Stroke 2010;41:1013-17

6. Morales $\mathrm{H}, \mathrm{Lu} \mathrm{A}, \mathrm{Kurosawa} \mathrm{Y}$, et al. Isomolar contrast causes smaller infarcts than low-osmolar contrast. In: Proceedings of the 48th American Society of Neuroradiology and Neuroradiology Education and Research Foundation Symposium, Boston, Massachusetts. May 15-20, 2010

7. Pfefferkorn T, Rosenberg GA. Closure of the blood-brain barrier by matrix metalloproteinase inhibition reduces rt-PA-mediated mortality in cerebral ischemia with delayed reperfusion. Stroke 2003;34:2025-30

T. Tomsick

University of Cincinnati Medical Center Cincinnati, Ohio

http://dx.doi.org/10.3174/ajnr.A3824 